\title{
One-Year Outcomes of Laparoscopic Sleeve Gastrectomy in Morbidly Obese Patients Regarding Preoperative BMI, Gender, and Age
}

Mohsen Tabasi

Pasteur Institute of Iran

Marziye Farsimadan

University of Guilan

Hamidreza Iranpour

Pasteur Institute of Iran

Fezzeh Elyasinia

Tehran University of Medical Sciences

Mohammad Reza Yazdannasab

Pasteur Institute of Iran

Seyed Davar Siadat

Pasteur Institute of Iran

Ahmadreza Soroush ( $\nabla$ soroush11344@gmail.com )

Tehran University of Medical Sciences

\section{Research Article}

Keywords: LSG, ASMBS, bleeding, pain, anastomotic leaks, Bariatric surgery (BS)

Posted Date: March 10th, 2021

DOl: https://doi.org/10.21203/rs.3.rs-288558/v1

License: (c) (1) This work is licensed under a Creative Commons Attribution 4.0 International License.

Read Full License 
One-Year Outcomes of Laparoscopic Sleeve Gastrectomy in Morbidly Obese Patients Regarding Preoperative BMI, Gender, and Age

\author{
Mohsen Tabasi ${ }^{a, b}$, Marziye Farsimadanc, , Hamidreza Iranpoor ${ }^{a, d}$, Fezzeh Elyasinia $^{\mathrm{e}}$, \\ Mohammad Reza Yazdannasab,g, Seyed Davar Siadat f,g*, Ahmadreza Soroush,** \\ ${ }^{a}$ Department of Molecular Biology, Pasteur Institute of Iran, Pasteur Ave., Tehran, 13164, Iran. \\ ${ }^{\mathrm{b}}$ Legal Medicine Research Center, Legal Medicine Organization, Tehran, Iran. \\ ${ }^{c}$ Department of Biology, Faculty of Sciences, University of Guilan, Rasht/Iran. \\ ${ }^{\mathrm{d}}$ Laboratory of Regenerative Medicine \& Biomedical Innovations, Pasteur Institute of Iran, \\ Tehran, Iran. \\ ${ }^{\mathrm{e}}$ Department of Surgery, Tehran University of Medical Sciences, Tehran, Iran. \\ ${ }^{\mathrm{f}}$ Department of Mycobacteriology and Pulmonary Research, Pasteur Institute of Iran, Tehran, \\ Iran. \\ ${ }^{\mathrm{g}}$ Microbiology Research Centre (MRC), Pasteur Institute of Iran, Tehran, Iran. \\ ${ }^{\mathrm{h}}$ Obesity and Eating Habits Research Center, Endocrinology and Metabolism Clinical Sciences \\ Institute, Tehran University of Medical Sciences, Tehran, Iran.
}

\title{
Co-Corresponding authors:
}

Professor Ahmadreza Soroush, Obesity and Eating Habits Research Center, Endocrinology and Metabolism Clinical Sciences Institute, 5th Floor, Shariati Hospital, North Kargar Ave, Tehran, Post Code-1411413137, Iran.

E-mail: soroush1344@gmail.com

Professor Seyed Davar Siadat, Department of Mycobacteriology and Pulmonary Research, Pasteur Institute of Iran, Tehran, Iran; Microbiology Research Center (MRC), Pasteur Institute of Iran, Tehran, Iran.

E-mail: d.siadat@gmail.com.

Running title: complications after LSG regarding preoperative BMI, gender, and age.

\begin{abstract}
The outcome of laparoscopic sleeve gastrectomy (LSG), one of the most common bariatric surgery (BS) procedure, may differ depending on the patient's age, gender, preoperative BMI. In this study, we aimed to evaluate the efficacy of LSG technique on weight loss, obesity-associated comorbidities and complications in patients undergoing LSG over three months and one year, regarding their age, gender, and BMI. The LSG associated complications in participants regarding their preoperative BMI (<39.9 and >39.9), age (30-40 and 40-50), and gender were assessed after
\end{abstract}


3 and 12 months. Besides, the remission and improvement rates of comorbid conditions in patients were examined after 12 months of LSG. Our results showed that LSG led to a significant weight loss in the resolution of obesity-associated co-morbidities and different complications after one year. No significant difference was found in the improvement and the resolution of obesityassociated co-morbidities according to gender. Also, the class 3 patients had significantly higher rates of hearing loss after 12 months and had higher levels of depression and brittle nails three months after the surgery. The younger patients also showed a significantly higher percentage of dry skin, intolerance to bread, and menstrual disorder compared to the older group.

\section{Introduction:}

Obesity has emerged as an epidemic that presents an unprecedented public health challenge ${ }^{1}$. Over the last decades, the prevalence of obesity has rapidly increased worldwide. Approximately, a third of the world's population is now classified as overweight or obese ${ }^{2}$.

The World Health Organization (WHO) defines overweight and obesity as abnormal or excessive fat accumulation that poses a risk to health ${ }^{3}$. Obesity is often misinterpreted by the public to be caused by lack of will leading to inappropriate dietary choices and physical inactivity. Indeed, obesity is a complicated chronic medical condition with remarkable negative impacts on human health and is caused by environmental, humoral, behavioral, and genetic factors ${ }^{1}$.

Obesity negatively influences almost all physiological functions of the body and is correlated to a wide variety of complications and comorbidities. It increases the risk of developing multiple disease conditions, such as type 2 diabetes mellitus, fatty liver disease, hypertension, myocardial infarction, stroke, dementia, osteoarthritis, obstructive sleep apnea, and several cancers, thereby contributing to adversely affect the quality of life, life expectancy, work productivity, and healthcare costs ${ }^{4}$. Additionally, several investigations have shown how obesity considerably affects patients psychologically and may lead to increased rates of mental disorders such as poor self-esteem and depression among them ${ }^{5}$. 
Bariatric surgery (BS) has become the most lasting and effective treatment for obesity as it dramatically leads to loss of fat mass and basically alleviates obesity-associated disorders ${ }^{1}$. Laparoscopic sleeve gastrectomy (LSG), one of the most common BS procedures is rather a safe and simple technique with promising short-term and midterm efficacy for severe obesity and type 2 diabetes that has gained great popularity over the last years ${ }^{6,7}$.

Aside from the degree of inherent risk with any surgery, LSG can result in a wide range of physical and mental complications ${ }^{8}$. According to the American Society for Metabolic and Bariatric Surgery (ASMBS) Clinical Issues Committee statement, the total complication rate for LSG is 0 $24 \%{ }^{9}$.

Postoperative complications can range from chronic to potentially life-altering issues. Acute complications such as bleeding, pain, anastomotic leaks, and blood clots are occur shortly postoperative. On the other hand, some patients may experience gastrointestinal issues such as food intolerance, dyspepsia, nausea, diarrhea, etc. as a complication of LSG. While these may appear immediately postoperative, some patients may experience them for an extended period ${ }^{10,11}$. The present study is one of the large sample size studies examining the complications after LSG. To better understand the impact of different factors including, preoperative BMI, gender, and age on the outcomes of LSG, the present study aimed to evaluate common after-surgery complications following LSG procedures in patients regarding their age, gender, and BMI. To the best of our knowledge, this is the first study to examine the outcomes of LSG based on the patient's age, gender, and preoperative BMI.

\section{Results}

\section{Preoperative characteristics}

Within the study period (from 2018 to 2020), 135 morbidly obese patients underwent LSG as a primary procedure for obesity treatment. From 135 patients that had completed 1 year of surgery, $126(82.3 \%)$ were available for follow-up. The sociodemographic characteristics of men and women included in this study are shown in Table 1. Among the participants, 30 (23.8\%) were men and $96(76.1 \%)$ were women. Nine patients were lost to follow-up primarily due to change of residence. Preoperative mean age was $37.43 \pm 6.15$ for men and $37.54 \pm 6.46$ years for women ( $\mathrm{p}=0.92)$. Mean height $(172.77 \pm 8.54$ vs. $169.70 \pm 7.30, \mathrm{p}=0.08)$, weight $(142.14 \pm 24.56$ vs. 
115.62 $\pm 20.51, \mathrm{p}=0.00)$, and mean BMI $(44.57 \pm 5.87$ vs. $42.65 \pm 5.01 \mathrm{~kg} / \mathrm{m} 2, \mathrm{p}=0.06)$ were comparable between the two populations.

Table 2 shows a summary of comorbidities and distribution of risk factors among the studied groups. Hyperlipidemia was the most frequent comorbidity in females compared to males $(80 \%$ vs. $80 \%, \mathrm{p}=0.70)$, followed by hypertension ( $36 \%$ vs. $35 \%, \mathrm{p}=0.78)$, hypothyroidism ( $13 \%$ vs. $25 \%, \mathrm{p}=0.11)$, depression ( $3 \%$ vs. $24 \%, \mathrm{p}=0.007)$, degenerative joint pain $(16 \%$ vs. $20 \%, \mathrm{p}=0.14)$, T2DM (insulin-dependent, 6\% vs. 6\%, p=0.95; non-insulin-dependent, 13\% vs. 13\%, p=0.80), obstructive sleep apnea (13\% vs. $10 \%, \mathrm{p}=0.81)$, and stomach ulcer (6 vs. $10 \%, \mathrm{p}=0.44)$ (Table 2$)$. There was also a significant difference in the prevalence of kidney stone (20\% vs. 5\%, p=0.02) and gallstone $(0 \%$ vs. $11 \%, \mathrm{p}=0.04)$ between the males and females.

\section{Type two diabetes mellitus, pre and post-LSG}

Of the 13 female patients with T2DM, nine were on metformin treatment, one patient was on metformin and insulin, and three patients were on diet therapy. Among male patients, three were on metformin treatment; one patient was on diet therapy. At 1 year postoperatively, only three (3/13) diabetic female patients remained on hypoglycemic therapy ( 2 patients on metformin and 1 patient on insulin) and one diabetic male had to keep taking metformin.

\section{Hypertension and hyperlipidemia, pre and post-LSG}

At the preoperative evaluation, there were no significant differences in the prevalence of these comorbidities among male and female groups (Table 2). After one year, hypertension resolved in $60 \%$ of cases, in particular, in $63 \%$ (7/11) of male patients and 58\% (20/34) of female patients. Hyperlipidemia resolved in $71 \%$ of patients: $79 \%$ (18/24) of male patients and $59 \%(59 / 77)$ of female patients.

\section{Obstructive sleep apnoea and degenerative joint pain, pre and post-LSG}

No significant differences in the frequency of obstructive sleep apnoea and degenerative joint pain were revealed among male and female groups (Table 2). After 12 months, obstructive sleep apnoea resolved in $7 \%$ of cases $(0 \%(0 / 4)$ of male patients and in $10 \%(1 / 10)$ of female patients) and degenerative joint pain was improved in $4 \%$ of patients $(0 \%(0 / 5)$ of male patients and $5 \%(1 / 19)$ of female patients). 


\section{Weight loss after LSG}

Three months and one year after LSG, a significant reduction was seen in the BMI of patients. After three months the BMI of patients decreased to 33.88 \pm 5.12 and after one year it significantly dropped to 29.54 \pm 4.41 . For females, the mean BMI dropped to $34.09 \pm 5.09$ and $29.10 \pm 4.40$ in three and one years after surgery, respectively. However, the mean BMI for males was 36.32 \pm 5.26 and 30.57 \pm 3.98 after three months and one year, respectively. A statically significant difference was found in the reduction of BMI between men and women after three and one years after the surgery $(\mathrm{P}=0.00)$.

Also, the mean BMI of patients aged between 30-40 was $44.17 \pm 5.20$ and that decreased to $34.35 \pm 5.54$ after three months and after a year it was 29.90 \pm 4.89 . For patients aged between 4050, the mean BMI was 43.80 \pm 5.96 and reduced to 35.12 \pm 4.56 after three months and to $30.65 \pm 3.72$ after a year. A statically significant difference was found in the reduction of BMI between younger and older patients after three and one years after the surgery $(\mathrm{P}=0.00)$.

\section{LSG-related complications regarding preoperative BMI, gender, and age}

The rates of complications in morbidly obese patients 3 and 12 months after LSG are shown in Table 3. Overall, the LSG-related complications were significantly decreased in 12 months compared to 3 months follow up after LSG. Nevertheless, hearing loss and depression increased in 12 months compared to 3 months follow up after LSG.

The postoperative data and common complications among the studies group regarding their preoperative BMI (<39.9 and >39.9) are summarized in Table 4. Our results indicated that the patients during three months and one year showed a significant decrease in the frequency of different complications investigated in this study including constipation, abdominal pain, dysphagia, depression, brittle nails, dry skin, hair loss, fatigue, menstrual disorder, food intolerance to pasta, bread, rice, meat, vegetable, and fruit, and their taste for sour and salty food; however, changes in the frequency of abdominal pain, depression, brittle nails, dry skin, hair loss, and menstrual disorder among patients were more noticeable than the other complications during three and twelve months after surgery. It is also noteworthy that the prevalence of complications including vision loss, hearing loss, severe hair loss, vomiting, and palpitations within the first hour after a meal decreased, but it was not significant. 
A sub-analysis was done according to the BMI of patients to investigate changes in the different complications among class 2 and class 3 obese patients during this period. Of 126 morbidly obese patients in this study, 33 and 93 participants had a preoperative BMI higher and lower than 39.9, respectively. Our analysis showed that class 3 patients had significantly higher rates of hearing loss after 12 months and had higher levels of depression and brittle nails three months after the surgery compared to class 2 obese patients. Also, the frequency of intolerance to pasta and meat was significantly increased among patients who had >39.9 BMI in comparison to the other group.

A sub-analysis considering the gender of patients was also performed (Table 5). The study group consisted of 30 men and 96 women and the analysis of the data revealed that female patients had significantly higher rates of dysphagia, brittle nails, and severe hair loss compared to male patients after 1 year of LSG. Moreover, their intolerance to pasta and fruits was significantly higher compared to males after three months. The number of women with the sweet and sour taste was also statistically higher than men.

Our sub-analysis according to the age of patients (Table 6) demonstrated that younger patients (3040 age group including 78 patients) had a significantly higher percentage of dry skin, intolerance to bread, and menstrual disorder compared to the older group (40-50 age group including 48 patients) three months after the LSG surgery and developed sweet taste increasingly after a year of surgery compared older patients. No significant differences were observed in the evaluation of other complications between these two age groups. Comorbid conditions remission and improvement rates were evaluated in patients after 12 months of LSG showing positive outcomes in remission and substantial improvement rate in comorbidity (Table 7).

\section{Discussion}

The outcome of LSG in patients undergoing BS treatment may differ depending on the patient's age, gender, preoperative BMI. Data from this study documented the efficacy of LSG technique on weight loss, obesity-associated co-morbidities and complications in patients undergoing LSG as a solo bariatric procedure over three months and one year, regarding their age, gender, and BMI. It is interesting to note that our results showed that LSG led to a significant weight loss, promising results in remission, and a considerable improvement in the resolution of obesity-associated comorbidities like T2DM, hypertension, and hyperlipidemia, and also in different complications including constipation, abdominal pain, dysphagia, depression, brittle nails, dry skin, hair loss, 
fatigue, and menstrual disorder after one year. No significant difference was found in the improvement and the resolution of obesity-associated co-morbidities according to gender; however, female patients had significantly higher rates of dysphagia, brittle nails, and severe hair loss compared to male patients after 1 year of LSG. Additionally, the class 3 patients had significantly higher rates of hearing loss after 12 months and had higher levels of depression and brittle nails three months after the surgery compared to class 2 obese patients. The younger patients also had a significantly higher percentage of dry skin, intolerance to bread, and menstrual disorder compared to the older group three months after the LSG surgery. To the best of our knowledge, this is the first study reporting the outcomes of LSG depending on the patient's age, gender, and preoperative BMI for three months and one year.

The actual approved indications for bariatric surgery are based on consensus guidelines issued by the National Institute of Health (NIH). They suggest surgery for people with a BMI of over 40 and people with a BMI of 35 or more who have at least two related comorbidities, according to this consensus. As a matter of fact, LSG may be the best option for patients with BMI between 35-40 who does not have two related comorbidities ${ }^{12}$.

BS has been considered as the most effective treatment for obesity and LSG, a frequently used BS technique, has gained great popularity among people over the last years, owing to its low morbidity and fast return to social life. The durability of weight loss, improvement in co-morbidities, and associated risk of mortality and morbidity are the main factors that account for the efficacy of a BS. Different LSG investigations showed a considerable resolution or improvement in comorbid conditions in patients with different age groups ${ }^{13-15}$, and also in high-risk surgical patients after a short ${ }^{16}$, medium- and long-term period ${ }^{17-19}$; however, the results were varying.

In line with previous studies, Sibier et al. in a study including 68 patients undergoing LSG realized that co-morbidities among patients improved considerably after five years and reported remission of type 2 diabetes among them at $85 \%{ }^{14}$. A similar pattern of results was obtained in a large study evaluating the long-term effects of LSG on 1050 patients, of whom $72.86 \%$ were women. The improvement or remission of comorbidities such as type 2 diabetes, hypertensive, and sleep apnea syndrome was found $57.2 \%, 19.2 \%$, and $85 \%$, respectively ${ }^{15}$. Similarly, the long-term efficacy of LSG among 96 patients from New Zealand showed significant improvement and resolution rates for type 2 diabetes (79\%), hypertension (61\%), and obstructive sleep apnea (73\%) among 
participants. They reported that weight loss outcomes at 5 year follow-up were modest ${ }^{20}$. In a relatively large study including 424 obese patients in India, complete improvement in obstructive sleep apnea, 69.3\% improvement in hypertension, 75\% improvement in hypothyroidism was shown after $\mathrm{LSG}^{21}$.

Kikkas and colleagues in a recent investigation have shown that LSG ensured long-term excess weight loss $61.0 \%$ at 5 years. They concluded that laparoscopic sleeve gastrectomy has a longterm effect on the significant improvement of triglycerides, low-density lipoproteins, and highdensity lipoproteins, but not on total cholesterol levels ${ }^{22}$. Considerable effects of LSG on different comorbidities were also shown in another study from Spain and the authors of this study introduced LSG to be safe and highly effective in terms of weight loss, particularly in patients with a preoperative BMI lower than $40 \mathrm{~kg} / \mathrm{m}^{2} 23$.

Khidir et al. in interesting research indicated the comparable weight loss results among patients based on their age and showed that LSG could lead to better improvement in younger patients ${ }^{24}$. Likewise, Angrisani et al. in a study consisted of 105 patients undergoing LSG over five years considered LSG to be an effective procedure at the long-term, with good weight loss outcomes and with a considerable improvement of obesity-associated co-morbidities. They concluded that younger age had a significantly higher percentage of total weight loss over 5 years ${ }^{25}$. Similar results were observed in another study in Germany. The authors of this study reported that LSG can be performed in younger ages without mortality and resolution rate of comorbidities and also weight loss was significantly higher compared to older ages ${ }^{26}$. As opposed to these studies, Woźniewska et al. in a very recent study in Poland showed significant weight loss in all patients to be independent of age and all individuals $(<45$ and $\geq 45$ years old) presented significant weight loss at 24 months after the surgery. However, the improvement in lipid and carbohydrate profile was rather faster and stronger in the older group compared to younger patients ${ }^{27}$.

The safety and effectiveness of LSG were frequently demonstrated in different studies ${ }^{28-32}$; however, some studies observed the severity of complications to be higher after LSG than other bariatric procedures ${ }^{33-35}$.

In conclusion, this study further supports the evidence of LSG being an effective technique for substantial and sustainable weight loss in morbidly obese patients. LSG provides promising results in remission and a considerable improvement in the resolution of different obesity-associated co- 
morbidities and different after surgery complications. With a significant weight loss at 1 year, resolution of co-morbidities, and less harsh complications after surgery among patients who chose LSG treatment, LSG is probably the procedure of choice in the management of obese patients, especially in developing countries where the rates of obesity are rather high among people. Our study emphasizes the importance and necessity of more research into the factors influencing the outcome of LSG like age, gender, and BMI. Further investigations with a large group of patients and better long-term follow-up are needed.

\section{Materials and Methods}

\section{Patients' selection}

A total of 126 morbidly obese patients who underwent LSG were enrolled in this longitudinal study from September 2018 to January 2020. All the participants were thoroughly informed about the project and procedures before providing written informed consent. Moreover, inclusion and exclusion criteria were defined according to our previous study ${ }^{7}$.

\section{Preoperative measures}

All patients were recommended to go on a low-carbohydrate high-protein diet at least a 2-week pre-operative. Sociodemographic characteristics, underlying medical history, obesity associated co-morbidities, and distribution of risk factors in morbidly obese patients were collected based on a standard questionnaire and general medical examination.

\section{Postoperative measures}

In this study, a "Postoperative measure" is defined as any LSG associated complications that occur 3 and 12 months after LSG in participants regarding their preoperative BMI (<39.9 and >39.9), age (30-40 and 40-50), and gender (male and female). Furthermore, we evaluated the remission (disappearance of the signs and symptoms of comorbidity) and improvement (the process that comorbidity moves toward the normal state) rate of comorbid conditions in patients after 12 months of LSG.

\section{Statistical analysis}

Data analysis was performed with the Stata software (v. 14). Categorical variables were presented as frequency (percentage) and continuous variables were summarized using median (min-max) or 
mean \pm standard deviation. The chi-square test was used to compare categorical variables, while Student's t-test was used to compare continuous variables. Differences between the groups before and 3 months after the surgery as well as 3 and 12 post-surgery were assessed using t-test and Fisher exact test. To check for normality, Kolmogorov-Smirnov test was used and Mann Whitney $\mathrm{U}$ test was used for non-normally distribution data. A p-value less than $0.05(\mathrm{p} \leq 0.05)$ was considered statistically significant.

\section{Confirmation statement}

Each listed author is submitting the paper in their own personal, professional capacity, and are not employees of an US-sanctioned government.

\section{Declarations}

\section{Ethics approval and consent to participate}

This study was conducted under the principles of the 1964 Helsinki declaration and its later amendments or comparable ethical standards. All the participants were thoroughly informed about the study and procedures before signing consent forms. Participants were assured of anonymity and confidentiality. The Research Ethics Committee of the Pasteur Institute of Iran, Tehran, approved this study (IR.PII. REC.1397.029).

Availability of data and materials: All data generated or analyzed in this study are included in the present article.

Competing interests: The authors have no conflicts of interest.

Funding: This project was financially supported by the Pasteur Institute of Iran (grant no. TP9567).

\section{Authors' Contribution:}

MT, MF, and HI writing paper and data analysis. MRY and FE data collecting. ARS and SDS conceptualization, Investigation, Formal analysis, Writing-review \& editing. All co-authors commented on the manuscript. All authors read and approved the final manuscript. 


\section{Acknowledgments}

This article was part of the project conducted by Mohsen Tabasi to fulfill the requirement for a Ph.D. degree. We would like to express our gratitude to the Pasteur Institute of Iran and Legal Medicine Research Center for providing financial support. We also would like to express our appreciation to the staff in Shariati Hospital of Tehran, Iran for their technical assistance. 


\section{References:}

1 Kheirvari, M. et al. The advantages and disadvantages of sleeve gastrectomy; clinical laboratory to bedside review. Heliyon 6, e03496 (2020).

2 Seidell, J. C. \& Halberstadt, J. The global burden of obesity and the challenges of prevention. Annals of Nutrition and Metabolism 66, 7-12 (2015).

3 Ofori-Asenso, R., Agyeman, A. A., Laar, A. \& Boateng, D. Overweight and obesity epidemic in Ghana - a systematic review and meta-analysis. BMC public health 16, 1239 (2016).

4 Blüher, M. Obesity: global epidemiology and pathogenesis. Nature Reviews Endocrinology 15, 288-298 (2019).

5 Casagrande, M., Boncompagni, I., Forte, G., Guarino, A. \& Favieri, F. Emotion and overeating behavior: Effects of alexithymia and emotional regulation on overweight and obesity. Eating and Weight Disorders-Studies on Anorexia, Bulimia and Obesity 25, 1333-1345 (2020).

6 Tabasi, M. et al. Gut Microbiota and Serum Biomarker Analyses in Obese Patients Diagnosed with Diabetes and Hypothyroid Disorder. Metabolic Syndrome and Related Disorders (2020).

7 Tabasi, M. et al. Modulation of the Gut Microbiota and Serum Biomarkers After Laparoscopic Sleeve Gastrectomy: a 1-Year Follow-Up Study. Obesity Surgery, 1-8.

8 Tabasi, M. et al. Positional Vertigo and Unilateral Gradual Hearing Loss Following Sleeve Gastrectomy: A Case Report. Diabetes, Metabolic Syndrome and Obesity: Targets and Therapy 13, 387 (2020).

9 of the American, C. I. C. \& for Metabolic, S. Sleeve gastrectomy as a bariatric procedure. Surgery for Obesity and Related Diseases 3, 573-576 (2007).

10 Sarkhosh, K., Birch, D. W., Sharma, A. \& Karmali, S. Complications associated with laparoscopic sleeve gastrectomy for morbid obesity: a surgeon's guide. Canadian journal of surgery 56, 347 (2013).

11 Campanile, F. C. et al. Acute complications after laparoscopic bariatric procedures: update for the general surgeon. Langenbeck's archives of surgery 398, 669-686 (2013).

12 Panel, N. I. o. H. C. D. Gastrointestinal surgery for severe obesity. Ann Intern Med 115, 956-961 (1991).

13 Abbas, M. et al. Outcomes of laparoscopic sleeve gastrectomy and Roux-en-Y gastric bypass in patients older than 60. Obesity surgery 25, 2251-2256 (2015).

14 Sieber, P. et al. Five-year results of laparoscopic sleeve gastrectomy. Surgery for Obesity and Related Diseases 10, 243-249 (2014).

15 Nocca, D. et al. Five-year results of laparoscopic sleeve gastrectomy for the treatment of severe obesity. Surgical endoscopy 31, 3251-3257 (2017).

16 Magee, C. J. et al. Laparoscopic sleeve gastrectomy for high-risk patients: weight loss and comorbidity improvement - short-term results. Obesity surgery 21, 547-550 (2011).

17 Srinivasa, S. et al. Early and mid-term outcomes of single-stage laparoscopic sleeve gastrectomy. Obesity surgery 20, 1484-1490 (2010).

18 Yormaz, S., Y1lmaz, H., Ece, I., Y1lmaz, F. \& Sahin, M. Midterm clinical outcomes of antrum resection margin at laparoscopic sleeve gastrectomy for morbid obesity. Obesity surgery 27, 910916 (2017).

19 Misra, S. et al. Long-term outcomes of laparoscopic sleeve gastrectomy from the Indian subcontinent. Obesity Surgery 29, 4043-4055 (2019).

20 Lemanu, D. P. et al. Five-year results after laparoscopic sleeve gastrectomy: a prospective study. Surgery for Obesity and Related Diseases 11, 518-524 (2015). 
21 Garg, H. et al. Mid to long term outcomes of Laparoscopic Sleeve Gastrectomy in Indian population: 3-7 year results-A retrospective cohort study. International Journal of Surgery 48, 201-209 (2017).

22 Kikkas, E. et al. Five-year outcome of laparoscopic sleeve gastrectomy, resolution of comorbidities, and risk for cumulative nutritional deficiencies. Scandinavian Journal of Surgery 108, 10-16 (2019).

23 Hoyuela, C. Five-year outcomes of laparoscopic sleeve gastrectomy as a primary procedure for morbid obesity: a prospective study. World journal of gastrointestinal surgery 9, 109 (2017).

24 Khidir, N. et al. Five-year outcomes of laparoscopic sleeve gastrectomy: a comparison between adults and adolescents. Obesity surgery 28, 2040-2045 (2018).

25 Angrisani, L. et al. Five-year results of laparoscopic sleeve gastrectomy: effects on gastroesophageal reflux disease symptoms and co-morbidities. Surgery for Obesity and Related Diseases 12, 960-968 (2016).

26 Benedix, F. et al. Perioperative course, weight loss and resolution of comorbidities after primary sleeve gastrectomy for morbid obesity: are there differences between adolescents and adults? Obesity surgery 27, 2388-2397 (2017).

27 Woźniewska, P. et al. The influence of patient's age on metabolic and bariatric results of laparoscopic sleeve gastrectomy in 2-year observation. BMC surgery 20, 1-8 (2020).

28 Hans, P. K., Guan, W., Lin, S. \& Liang, H. Long-term outcome of laparoscopic sleeve gastrectomy from a single center in mainland China. Asian journal of surgery 41, 285-290 (2018).

29 Capoccia, D. et al. Long-term metabolic effects of laparoscopic sleeve gastrectomy. Obesity surgery 28, 2289-2296 (2018).

30 Golomb, I., David, M. B., Glass, A., Kolitz, T. \& Keidar, A. Long-term metabolic effects of laparoscopic sleeve gastrectomy. JAMA surgery 150, 1051-1057 (2015).

31 Abbatini, F. et al. Long-term effects of laparoscopic sleeve gastrectomy, gastric bypass, and adjustable gastric banding on type 2 diabetes. Surgical endoscopy 24, 1005-1010 (2010).

32 Todkar, J. S., Shah, S. S., Shah, P. S. \& Gangwani, J. Long-term effects of laparoscopic sleeve gastrectomy in morbidly obese subjects with type 2 diabetes mellitus. Surgery for Obesity and related diseases $\mathbf{6}, 142-145$ (2010).

33 Vitiello, A., Pilone, V., Ferraro, L. \& Forestieri, P. Is the sleeve gastrectomy always a better procedure? Five-year results from a retrospective matched case-control study. Obesity surgery 28, 2333-2338 (2018).

$34 \mathrm{Gu}$, L. et al. A meta-analysis of the medium-and long-term effects of laparoscopic sleeve gastrectomy and laparoscopic Roux-en-Y gastric bypass. BMC surgery 20, 30 (2020).

35 Golzarand, M., Toolabi, K. \& Farid, R. The bariatric surgery and weight losing: a meta-analysis in the long-and very long-term effects of laparoscopic adjustable gastric banding, laparoscopic Rouxen-Y gastric bypass and laparoscopic sleeve gastrectomy on weight loss in adults. Surgical endoscopy 31, 4331-4345 (2017). 


\begin{tabular}{|c|c|c|c|c|c|}
\hline Parameters & Male $(\mathrm{N}=30)$ & Female $(\mathrm{N}=96)$ & Total $(\mathrm{N}=126)$ & P-value & \\
\hline Age (mean \pm SD) & $37.43 \pm 6.15$ & $37.54 \pm 6.46$ & $37.45 \pm 6.21$ & 0.92 & \\
\hline Height $(\mathrm{cm})$ & $172.77 \pm 8.54$ & $169.70 \pm 7.30$ & $170.08 \pm 9.53$ & 0.08 & \\
\hline Weight (kg) & $142.14 \pm 24.56$ & $115.62 \pm 20.51$ & $122.50 \pm 24.42$ & 0.00 & \\
\hline BMI $(\mathrm{kg} / \mathrm{m} 2)$ & $44.57 \pm 5.87$ & $42.65 \pm 5.01$ & $43.15 \pm 5.29$ & 0.06 & \\
\hline Smoking & $18(60)$ & $29(30)$ & $47(37)$ & 0.017 & \\
\hline Coffee consumption & 11(36) & $42(44)$ & $53(42)$ & 0.22 & \\
\hline Alcohol consumption & $10(33)$ & $18(19)$ & $28(22)$ & 0.18 & \\
\hline \multicolumn{6}{|l|}{ Medications } \\
\hline Metformin & $4(13)$ & $14(14)$ & $18(14)$ & 0.86 & \multirow{9}{*}{$\begin{array}{rr}\text { Table } & 1 . \\
\text { Sociodemographic } \\
\text { and } & \text { clinical }\end{array}$} \\
\hline Levothyroxine & $4(13)$ & $25(26)$ & $29(23)$ & 014 & \\
\hline Insulin & $1(3)$ & $5(5)$ & $6(4)$ & 0.67 & \\
\hline Atorvastatin & $3(10)$ & $8(8)$ & $11(9)$ & 0.77 & \\
\hline Metoral & $3(10)$ & $4(4)$ & $7(5)$ & 0.22 & \\
\hline Marital status & & & & & \\
\hline Single & $12(40)$ & $26(27)$ & $38(30)$ & 0.17 & \\
\hline Married & $17(56)$ & $67(69)$ & $84(66)$ & 0.18 & \\
\hline Divorced & $1(3)$ & $3(3)$ & $4(3)$ & 0.95 & \\
\hline \multicolumn{5}{|l|}{ Educational level } & \multirow{10}{*}{$\begin{array}{l}\text { characteristics of } \\
\text { morbidly obese } \\
\text { patient candidates } \\
\text { for LSG. }\end{array}$} \\
\hline School level & $13(43)$ & $42(44)$ & $55(43)$ & 0.96 & \\
\hline University level & $17(56)$ & $54(56)$ & $71(56)$ & 0.94 & \\
\hline \multicolumn{5}{|l|}{ Geographical area } & \\
\hline Urban & 193) & $7(7)$ & $26(20)$ & 0.00 & \\
\hline Rural & $29(96)$ & $89(92)$ & $118(93)$ & 0.43 & \\
\hline \multicolumn{5}{|l|}{ Occupation } & \\
\hline Government's employee & $16(53)$ & $39(40)$ & $55(43)$ & 0.22 & \\
\hline Homemaker & $0(0)$ & 47(49) & $47(37)$ & 0.00 & \\
\hline Self employed & $18(60)$ & $0(0)$ & $18(14)$ & 0.00 & \\
\hline
\end{tabular}


Table 2. Prevalence of obesity associated co-morbidities and distribution of risk factors in morbidly obese patient candidates for LSG.

\begin{tabular}{|c|c|c|c|c|}
\hline Clinical status & Male $(\mathrm{N}=30)$ & Female $(\mathrm{N}=96)$ & Total $(\mathrm{N}=126)$ & P-value \\
\hline \multicolumn{5}{|l|}{ Diabetes } \\
\hline Type 1 & $2(6)$ & $6(6)$ & $8(6)$ & 0.95 \\
\hline Type 2 & $4(13)$ & 13(13) & 17(13) & 0.80 \\
\hline Non- diabetic & $29(96)$ & $81(84)$ & $110(87)$ & 0.90 \\
\hline Hypertension & $11(36)$ & $34(35)$ & $45(35)$ & 0.78 \\
\hline Hyperlipidemia & $24(80)$ & $77(80)$ & $101(80)$ & 0.70 \\
\hline Obstructive sleep apnea & $4(13)$ & $10(10)$ & $14(11)$ & 0.81 \\
\hline Degenerative joint pain & $5(16)$ & $19(20)$ & $r 4(19)$ & 0.14 \\
\hline History of depression & $1(3)$ & $23(24)$ & $24(19)$ & 0.007 \\
\hline History of gallstone & $0(0)$ & 11(11) & $11(8)$ & 0.04 \\
\hline History of kidney stone & $6(20)$ & $5(5)$ & $11(8)$ & 0.02 \\
\hline Stomach ulcer caused by $H$. pylori & $2(6)$ & $10(10)$ & $12(8)$ & 0.44 \\
\hline \multicolumn{5}{|l|}{ History of bariatric } \\
\hline Abdominal liposuction & $0(0)$ & $2(2)$ & $2(1)$ & 0.39 \\
\hline Intragastric balloon & $2(6)$ & $3(3)$ & $5(4)$ & 0.46 \\
\hline Abdominal lipomatics & $0(0)$ & $8(8)$ & $8(6)$ & 0.08 \\
\hline Abdominoplasty & $0(0)$ & 4(4) & 4(3) & 0.23 \\
\hline None & $28(93)$ & $85(88)$ & 113(89) & 0.15 \\
\hline \multicolumn{5}{|l|}{ Hormonal abnormalities } \\
\hline Hypothyroidism & $4(13)$ & $24(25)$ & $28(22)$ & 0.11 \\
\hline Testosterone disorder & $0(0)$ & $2(2)$ & $2(1)$ & 0.39 \\
\hline Menstrual disorder & $0(0)$ & $4(4)$ & $4(3)$ & 0.23 \\
\hline Hypothyroidism and menstrual & $0(0)$ & $2(2)$ & $2(1)$ & 0.39 \\
\hline None & $26(86)$ & $68(70)$ & $94(74)$ & 0.018 \\
\hline \multicolumn{5}{|l|}{ Fatty liver } \\
\hline Grade 1 & $4(13)$ & $12(12)$ & $16(12)$ & 0.92 \\
\hline Grade 1 & $2(6)$ & $5(5)$ & $7(5)$ & 0.87 \\
\hline Grade 1 & $1(3)$ & $2(2)$ & $3(2)$ & 0.76 \\
\hline None & 27(89) & $81(84)$ & $108(86)$ & 0.62 \\
\hline \multicolumn{5}{|l|}{ Pregnancy history } \\
\hline Caesarean & - & $42(43)$ & $42(33)$ & - \\
\hline Natural & - & 11(11) & $11(8)$ & - \\
\hline Caesarean and natural & - & $13(12)$ & $13(10)$ & - \\
\hline None & & $34(35)$ & $34(27)$ & - \\
\hline \multicolumn{5}{|l|}{ Number of pregnancies } \\
\hline One pregnancy & - & $29(30)$ & $29(23)$ & - \\
\hline More than one pregnancy & - & $37(38)$ & $37(29)$ & - \\
\hline
\end{tabular}


Table 3. Rate (n/\%) of complications 3 and 12 months after LSG in morbidly obese patients.

\begin{tabular}{cccc}
\hline Complications & 3 months post-LSG [n (\%)] & 12 months post-LSG [n (\%)] & P-value \\
\hline Vision loss & $14(11)$ & $12(9)$ & 0.67 \\
\hline Hearing loss & $6(4)$ & $9(7)$ & 0.42 \\
\hline Constipation & $105(83)$ & $76(60)$ & $\mathbf{0 . 0 0}$ \\
\hline Abdominal pain & $93(74)$ & $22(17)$ & $\mathbf{0 . 0 0}$ \\
\hline Dysphagia & $86(68)$ & $57(45)$ & $\mathbf{0 . 0 0}$ \\
\hline Food intolerance to & & & $\mathbf{0 . 0 0}$ \\
Pasta & $48(30)$ & $9(7)$ & $\mathbf{0 . 0 0}$ \\
Bread & $112(88)$ & $41(32)$ & $\mathbf{0 . 0 0}$ \\
Rice & $125(99)$ & $48(38)$ & $\mathbf{0 . 0 0 2}$ \\
Meat & $26(20)$ & $9(7)$ & $\mathbf{0 . 0 0 6}$ \\
Vegetable & $14(11)$ & $3(2)$ & 0.12 \\
Fruit & $36(28)$ & $7(5)$ & $\mathbf{0 . 0 0}$ \\
Milk & $8(6)$ & $3(2)$ & $\mathbf{0 . 0 0}$ \\
\hline Depression & $9(7)$ & $52(41)$ & $\mathbf{0 . 0 0}$ \\
\hline Brittle nails & $63(50)$ & $23(18)$ & $\mathbf{0 . 0 0}$ \\
\hline Dry skin & $107(85)$ & $39(31)$ & 0.13 \\
\hline Hair loss & $100(79)$ & $21(16)$ & \\
\hline Severe hair loss & $26(20)$ & $17(13)$ & 0.16 \\
\hline Types of taste & & & $\mathbf{0 . 0 0 1}$ \\
Sweet & $51(40)$ & $62(49)$ & $\mathbf{0 . 0 2}$ \\
\hline Sour and salty & $56(44)$ & $30(24)$ & 0.07 \\
Normal & $19(15)$ & $34(27)$ & $\mathbf{0 . 0 0}$ \\
\hline Vomiting & $45(36)$ & $32(25)$ & $\mathbf{0 . 0 0}$ \\
\hline Fatigue & $77(61)$ & $47(37)$ & 0.17 \\
\hline Menstrual disorder & $67(53)$ & $28(22)$ & \\
\hline Palpitations within the & $33(26)$ & $24(19)$ & \\
first hour after a meal & & & \\
\hline & & & \\
\hline & & & \\
\hline & & & \\
\hline & & & \\
\hline
\end{tabular}




\begin{tabular}{|c|c|c|c|c|c|c|}
\hline \multirow[t]{2}{*}{ Complications } & \multicolumn{2}{|c|}{3 months post-LSG [n (\%)] } & \multicolumn{4}{|c|}{12 months post-LSG [n (\%)] } \\
\hline & $<39.9$ & $>39.9$ & P-value & $<39.9$ & $>39.9$ & P-value \\
\hline Vision loss & $4(12)$ & $10(11)$ & 0.83 & $2(6)$ & $10(11)$ & 0.43 \\
\hline Hearing loss & $1(3)$ & $5(5)$ & 0.58 & $5(15)$ & $4(4)$ & $\mathbf{0 . 0 3}$ \\
\hline Constipation & $28(85)$ & $77(83)$ & 0.78 & $16(48)$ & $60(64)$ & 0.10 \\
\hline Abdominal pain & $27(81)$ & $66(71)$ & 0.22 & $6(18)$ & $16(170$ & 0.89 \\
\hline Dysphagia & $22(66)$ & $64(69)$ & 0.82 & $15(45)$ & $42(45)$ & 0.77 \\
\hline \multicolumn{7}{|l|}{ Food intolerance to } \\
\hline Pasta & $11(33)$ & $37(40)$ & 0.51 & $5(15)$ & $4(4)$ & 0.03 \\
\hline Bread & $28(85)$ & $84(92)$ & 0.39 & $14(42)$ & $27(29)$ & 0.15 \\
\hline Rice & $33(100)$ & $92(99)$ & 0.00 & $16(48)$ & $32(34)$ & 0.15 \\
\hline Meat & $9(27)$ & $17(18)$ & 0.27 & $5(15)$ & $4(4)$ & 0.03 \\
\hline Vegetable & $3(9)$ & $11(12)$ & 0.66 & $2(6)$ & $1(1)$ & 0.10 \\
\hline Fruit & $9(27)$ & $27(29)$ & 0.84 & $2(6)$ & $5(5)$ & 0.88 \\
\hline Milk & $1(3)$ & $7(7)$ & 0.36 & $0(0)$ & $3(3)$ & 0.29 \\
\hline Depression & $5(15)$ & $4(4)$ & 0.03 & $12(36)$ & $40(43)$ & 0.50 \\
\hline Brittle nails & $21(7)$ & $42(45)$ & 0.05 & $4(12)$ & $19(20)$ & 0.28 \\
\hline Dry skin & $29(87)$ & $78(83)$ & 0.89 & $12(36)$ & $27(29)$ & 0.43 \\
\hline Hair loss & $26(78)$ & $74(24)$ & 0.92 & $8(24)$ & $13(14)$ & 0.17 \\
\hline Severe hair loss & $7(21)$ & $19(20)$ & 0.92 & $5(15)$ & $12(13)$ & 0.74 \\
\hline \multicolumn{7}{|l|}{ Types of taste } \\
\hline Sweet & $10(30)$ & $40(43)$ & 0.20 & $16(48)$ & $46(49)$ & 0.92 \\
\hline Sour and salty & $16(48)$ & $41(44)$ & 0.66 & $7(21)$ & $23(25)$ & 0.68 \\
\hline Normal & $7(21)$ & $12(13)$ & 0.25 & $9(27)$ & $24(26)$ & 0.86 \\
\hline Vomiting & $12(36)$ & $33(35)$ & 0.92 & $7(21)$ & $25(27)$ & 0.52 \\
\hline Fatigue & $16(48)$ & $61(65)$ & 0.08 & $10(30)$ & $37(39)$ & 0.33 \\
\hline Menstrual disorder & $19(57)$ & $48(51)$ & 0.55 & $5(15)$ & $23(24)$ & 0.25 \\
\hline $\begin{array}{l}\text { Palpitations within } \\
\text { the first hour after a } \\
\text { meal }\end{array}$ & $7(21)$ & $26(28)$ & 0.44 & $8(24)$ & $16(17)$ & 0.37 \\
\hline
\end{tabular}

Table 4.

Comparison of different complications 3 and 12 months after LSG in morbidly obese patients regarding their preoperative BMI $(<39.9$ and $>39.9$ ). 
Table 5. Frequency and comparison of different complications 3 and 12 months after LSG in morbidly obese patients regarding gender.

\begin{tabular}{|c|c|c|c|c|c|c|}
\hline \multirow[t]{2}{*}{ Complications } & \multicolumn{3}{|c|}{3 months post-LSG [n (\%)] } & \multicolumn{3}{|c|}{12 months post-LSG [n (\%)] } \\
\hline & Male & Female & P-value & Male & Female & P-value \\
\hline Vision loss & $5(16)$ & $9(9)$ & 0.26 & $4(13)$ & $8(8)$ & 0.41 \\
\hline Hearing loss & $2(6)$ & $4(41)$ & 0.57 & $0(0)$ & $9(9)$ & 0.08 \\
\hline Constipation & $22(30)$ & $83(86)$ & 0.09 & $17(56)$ & $59(61)$ & 0.64 \\
\hline Abdominal pain & $20(66)$ & $73(76)$ & 0.30 & $7(23)$ & $15(15)$ & 0.15 \\
\hline Dysphagia & $23(76)$ & $63(65)$ & 0.25 & $7(23)$ & $45(47)$ & 0.02 \\
\hline \multicolumn{7}{|l|}{ Food intolerance to } \\
\hline Pasta & $6(20)$ & $42(42)$ & 0.01 & 1(3) & $8(8)$ & 0.35 \\
\hline Bread & $29(96)$ & $83(86)$ & 0.12 & $8(26)$ & $33(34)$ & 0.49 \\
\hline Rice & $30(100)$ & $95(99)$ & 0.57 & $8(26)$ & $40(41)$ & 0.14 \\
\hline Meat & $5(16)$ & $21(22)$ & 0.53 & $2(6)$ & $7(7)$ & 0.90 \\
\hline Vegetable & $3(10)$ & 11(11) & 0.82 & $0(0)$ & $3(3)$ & 0.32 \\
\hline Fruit & $13(43)$ & $24(25)$ & 0.05 & $3(10)$ & $4(4)$ & 0.22 \\
\hline Milk & $1(3)$ & $7(7)$ & 0.43 & $1(3)$ & $2(2)$ & 0.69 \\
\hline Depression & $1(3)$ & $8(8)$ & 0.35 & $11(36)$ & $41(43)$ & 0.55 \\
\hline Brittle nails & $14(46)$ & $49(51)$ & 0.67 & $2(6)$ & $21(22)$ & 0.05 \\
\hline Dry skin & $25(83)$ & $82(85)$ & 0.78 & $7(23)$ & $32(33)$ & 0.30 \\
\hline Hair loss & $24(80)$ & $66(69)$ & 0.23 & $3(10)$ & $17(17)$ & 0.31 \\
\hline Severe hair loss & $3(10)$ & $23(24)$ & 0.09 & $1(3)$ & $16(16)$ & 0.05 \\
\hline \multicolumn{7}{|l|}{ Types of taste } \\
\hline Sweet & $17(56)$ & $33(34)$ & 0.02 & $14(46)$ & $48(50)$ & 0.75 \\
\hline Sour and salty & $8(26)$ & $48(50)$ & 0.02 & $6(20)$ & $24(25)$ & 0.57 \\
\hline Normal & $5(16)$ & $14(14)$ & 0.78 & $10(33)$ & $24(25)$ & 0.36 \\
\hline Vomiting & $9(30)$ & $36(37)$ & 0.45 & $5(16)$ & $27(28)$ & 0.20 \\
\hline Fatigue & $20(66)$ & $57(59)$ & 0.47 & $12(40)$ & $35(36)$ & 0.72 \\
\hline Menstrual disorder & $0(0)$ & $67(67)$ & - & $0(0)$ & $28(29)$ & - \\
\hline
\end{tabular}




\begin{tabular}{llllll}
\hline $\begin{array}{l}\text { Palpitations within the } \\
\text { first hour after a meal }\end{array}$ & $8(26)$ & $25(26)$ & 0.94 & $7(23)$ & $17(17)$ \\
\hline
\end{tabular}

Table 6. Frequency and difference of complications in morbidly obese patients 3 and 12 months after LSG regarding age.

\begin{tabular}{|c|c|c|c|c|c|c|}
\hline \multirow[t]{2}{*}{ Complications } & \multicolumn{3}{|c|}{3 months post-LSG [n (\%)] } & \multicolumn{3}{|c|}{12 months post-LSG [n (\%)] } \\
\hline & $20-30$ & $30-40$ & P-value & $20-30$ & $30-40$ & P-value \\
\hline Vision loss & $9(11)$ & $5(10)$ & 0.84 & $10(13)$ & $2(4)$ & 0.10 \\
\hline Hearing loss & $4(5)$ & $2(4)$ & 0.80 & $4(18)$ & $5(10)$ & 0.26 \\
\hline Constipation & $66(85)$ & $39(81)$ & 0.62 & $45(57)$ & $31(46)$ & 0.44 \\
\hline Abdominal pain & $55(70)$ & $38(79)$ & 0.28 & $6(20)$ & $6(12)$ & 0.37 \\
\hline Dysphagia & $52(66)$ & $34(71)$ & 0.62 & $35(45)$ & $22(46)$ & 0.91 \\
\hline Pasta & $29(37)$ & 19(39) & 0.78 & $4(18)$ & $5(10)$ & 0.26 \\
\hline Bread & $59(75)$ & 43(89) & 0.05 & $24(31)$ & $17(35)$ & 0.58 \\
\hline Rice & $77(98)$ & $48(100)$ & 0.43 & $30(38)$ & $18(37)$ & 0.91 \\
\hline Meat & $12(15)$ & $14(29)$ & 0.06 & $6(7)$ & $3(6)$ & 0.76 \\
\hline Vegetable & $8(10)$ & $6(12)$ & 0.69 & $3(3)$ & $0(0)$ & 0.16 \\
\hline Fruit & $23(29)$ & $13(27)$ & 0.77 & $4(4)$ & $3(6)$ & 0.79 \\
\hline Milk & $7(9)$ & $1(2)$ & 0.12 & $3(3)$ & $0(0)$ & 0.16 \\
\hline Brittle nails & $44(56)$ & $19(39)$ & 0.06 & $12(15)$ & $11(23)$ & 0.28 \\
\hline Dry skin & $71(91)$ & $36(75)$ & 0.01 & $28(36)$ & $11(23)$ & 0.12 \\
\hline Hair loss & $59(75)$ & $41(85)$ & 0.18 & $12(15)$ & $7(14)$ & 0.90 \\
\hline Severe hair loss & $19(24)$ & $7(14)$ & 0.18 & $9(11)$ & $8(16)$ & 0.41 \\
\hline \multicolumn{7}{|l|}{ Types of taste } \\
\hline Sweet & $40(51)$ & $17(35)$ & 0.08 & $45(57)$ & $17(35)$ & 0.01 \\
\hline Sour and salty & $31(40)$ & $25(52)$ & 0.17 & $15(19)$ & $15(31)$ & 0.12 \\
\hline Normal & $13(16)$ & $6(12)$ & 0.52 & $18(23)$ & $16(33)$ & 0.20 \\
\hline Vomiting & $27(34)$ & $18(37)$ & 0.74 & $24(31)$ & $8(16)$ & 0.07 \\
\hline Fatigue & $48(61)$ & $29(60)$ & 0.90 & $32(41)$ & $15(31)$ & 0.27 \\
\hline Menstrual disorder & $48(61)$ & 19(39) & 0.01 & $22(28)$ & $6(12)$ & 0.03 \\
\hline
\end{tabular}




$\begin{array}{lllll}\text { Palpitations within the } & 19(24) & 14(29) & 0.55 & 0.31 \\ \text { first hour after a meal } & & & 7(14)\end{array}$

Table 7. Remission and improvement of comorbid conditions in morbidly obese patients after 12 months of LSG.

\begin{tabular}{lcc}
\hline Comorbidity & $\begin{array}{c}\text { Remission/ } \\
\text { Comorbidity }\end{array}$ & $\begin{array}{c}\text { Improvement/ } \\
\text { Comorbidity }\end{array}$ \\
\hline $\begin{array}{l}\text { Type-2 } \\
\text { diabetes } \\
\text { mellitus }\end{array}$ & $9 / 14$ & $1 / 14$ \\
\hline Hypertension & $27 / 45$ & $6 / 45$ \\
\hline Hyperlipidemia & $67 / 101$ & $14 / 101$ \\
\hline $\begin{array}{l}\text { Obstructive } \\
\text { sleep apnea }\end{array}$ & $1 / 14$ & $3 / 14$ \\
\hline $\begin{array}{l}\text { Degenerative } \\
\text { joint pain }\end{array}$ & $1 / 24$ & $7 / 24$ \\
\hline
\end{tabular}

